\title{
An ab initio coupled cluster theory of quantum spin lattices and their quantum critical behaviour
}

\author{
By R. F. BISHOP and D. J. J. FARNELL \\ Department of Physics, UMIST, University of Manchester Institute of Science and \\ Technology, PO Box 88, Manchester M60 1QD, UK
}

\begin{abstract}
Strongly interacting quantum spin-lattice models exhibit a wide variety of phases with diverse and subtle magnetic ordering properties. Their detailed description within a unified microscopic framework poses a real challenge for the many-body theorist. By specific application to the spin- $-\frac{1}{2}$ anisotropic Heisenberg model on a square lattice, we show how the $a b$ initio coupled cluster method, which has already been very successfully applied to a wide variety of quantum many-body and field-theoretic systems, may be very efficiently and systematically implemented for spin-lattice models. Results for such local properties as the ground-state energy and sublattice magnetization are thereby obtained which are on a par with those from the best of the available alternative methods. Furthermore, we demonstrate explicitly how the coupled cluster method now also provides an effective and fully microscopic tool to yield systematic and accurate estimates of the zero-temperature quantum phase transition boundaries between states of different quantum order, as well as of the critical behaviour of the system in the vicinity of the transition points.
\end{abstract}

\section{Introduction}

In recent years the $a b$ initio techniques available for treating the properties of quantum-mechanical manybody systems at the fully microscopic level have become increasingly refined and accurate. The inexorable rise in the power of modern computers has also allowed the techniques to be applied to systems of ever-increasing complexity, whether these be larger or more complicated molecules or infinite systems with more subtle ordering properties.

One such method which stands to the fore in this regard is the coupled cluster method (CCM) [1-9] The CCM, already very well known to the quantum chemistry community $[2,8]$ has also become widely recognized throughout the theoretical physics community as providing one of the most powerful, most widely applicable, and most accurate at attainable levels of practical implementation, of all available $a b$ initio microscopic techniques of quantum many-body/field theory.

Despite the many achievements of the CCM and other techniques of modern quantum many-body theory (such as the method of correlated basis functions, which provides a systematic means of improving upon the variational results using such popular trial correlated wave functions as those used in Jastrow theory), the use of these techniques has been largely limited up until now to a local description of the properties of the system under consideration, rather than to its global behaviour. Thus, until very recently, most of these fully microscopic calculations had been restricted to calculations of such local properties as the ground-state energy, the excitation spectrum, and such other properties as the relevant order parameters and correlation functions. By contrast, very little progress had been made on using the same techniques to make contact at the microscopic level with the otherwise quite disparate corpus of work which relates to the study of phase transitions in infinite systems and to related (e.g. shape) transitions in finite systems. This situation is now beginning to change in fundamental ways, due largely to recent results obtained using the CCM which we describe in this article.

In order to set the scene for the results to be presented, we note that there are now many physical systems which are characterized by novel ground states which display quantum order in some regions of the relevant parameter space. Such regions are delimited by critical values which mark the respective quantum phase transitions. Very often, the critical phenomena displayed by the quantum systems differ profoundly from their corresponding classical counterparts (where they exist). The subtle correlated quantum-mechanical states usually cannot sensibly be viewed within the traditional language of Landau's theory of Fermi liquids, for example, or of other comparable phenomenological approaches which have been so useful in the past for so many conventional quantum many-body systems. Examples of systems or phenomena which fall into the unconventional (or novel) class include heavy fermions, the fractional quantum Hall effect, new quantum states in the condensed phases of helium, confinement/decon- 
finement phase transitions in gauge field theories, high-temperature superconductors and other strongly correlated electronic systems, and various phases of (antiferro)magnetic materials and low-dimensional quantum spin lattices.

The standard many-body techniques, such as perturbation theory, mean-field theories, and variational calculations, which have been successfully developed and applied for conventional systems, typically fail completely for these unconventional systems characterized by novel quantum order. One of the key challenges for modern quantum many-body theory is now to develop and exploit microscopic techniques which are capable of describing both these novel and the more conventional systems. A prime objective of our own recent work on quantum lattice systems [10-18] has been to show that at least one such modern technique, namely the CCM, is in fact already able to bridge these two classes of systems.

We note that the CCM has been applied over the last five or so years to a variety of lattice Hamiltonian systems $[15,16]$ including spin lattices of interest, for example, in magnetism [10-14, 19-26] and the solid phases of ${ }^{3} \mathrm{He}[19]$ electron lattice models of interest, for example, in the cuprate high-temperature superconductors (e.g. the Hubbard model) $[17,27\}$ and lattice gauge theories, such as the Abelian $U$ (1) model [18] and the non-Abelian $S U$ (2) model [28] In all cases the CCM may readily be implemented to high orders of approximation by the use of computer-algebraic techniques. Values for ground-state (and, increasingly, also excited-state) properties are thereby obtained which are fully competitive with those from other state-ofthe-art calculations, including the much more computationally intensive quantum Monte Carlo techniques. What we now further demonstrate, by a specific application to the spin- $\frac{1}{2}$ anisotropic Heisenberg model on the two-dimensional (2D) square lattice, is that the CCM can also provide information on the quantum order and quantum criticality inherent in this model. We believe that this example illustrates how the CCM is now well placed to study in a very systematic and unbiased manner the quantum phase transitions of the novel non-Fermi-liquid systems discussed above.

In section 2 below we briefly describe the fundamentals of the CCM as it is applied to quantum spin lattices, and in section 3 we describe its application in detail to various phases of the $2 \mathrm{D}$ spin- $-\frac{1}{2} X X Z$ (or anisotropic Heisenberg) model on a square lattice. The results are discussed in section 4, with special emphasis both on their accuracy and their ability to predict phase transitions and to describe the ensuing critical behaviour at the transition points. We conclude in section 5 with a discussion of possible extensions of the method and its potential for use with other systems which exhibit quantum phase transitions between states of different quantum order.

\section{The CCM for quantum spin lattices}

Although detailed descriptions of both the general CCM formalism [1-9] and its application to specific spin-lattice models [10-14] have been given elsewhere, we highlight here such of the essential general ingredients as are required for present purposes. Our aim in this section is to be as general as possible.

In the so-called single-reference version of the normal (as opposed to the extended [5, 7]) variant of the CCM, to which we restrict ourselves here, we first require the choice of a suitable single model or reference state $|\Phi\rangle$, in terms of which a quantitative and systematic description of the many-body (i.e. in the present case, multispin) correlations or fluctuations may be given. We defer until later the important question of how to choose $|\Phi\rangle$ in practice, but simply recall now that it is required only to be a cyclic vector with respect to two well-defined Abelian subalgebras of multi-configurational creation operators $\left\{C_{I}^{+}\right\}$and their Hermitian-adjoint destruction counterparts $\left\{C_{I}^{-} \equiv\left(C_{I}^{+}\right)^{\dagger}\right\}$. Thus, $|\Phi\rangle$ plays the role of a vacuum state with respect to a suitable set of (mutually commuting) many-body creation operators $\left\{C_{I}^{+}\right\}$,

$$
C_{I}^{-}|\Phi\rangle=0, \quad \forall l \neq 0,
$$

with $C_{0}^{-} \equiv I$, the identity operator. These operators are also complete in the many-body Hilbert (or Fock) space,

$$
I=|\Phi\rangle\left\langle\Phi\left|+\sum_{I \neq 0} C_{I}^{+}\right| \Phi\right\rangle \Phi \mid C_{I}^{-} .
$$

The choice of the operators $\left\{C_{I}^{+}\right\}$clearly depends on the choice of $|\Phi\rangle$, but for spin-lattice problems in general $C_{I}^{+}$will involve products of the basic $S U(2)$ spin operators $\left\{s_{k}^{+}, s_{k}^{-}, s_{k}^{z}\right\}$ on different lattice sites $k$, which obey the fundamental commutation relations,

$$
\left[\xi_{k}^{z}, s_{l}^{ \pm}\right]= \pm_{s_{k}}^{ \pm} \delta_{k l} ; \quad\left[s_{k}^{+}, s_{l}^{-}\right]=2 s_{k}^{z} \delta_{k l}
$$

where $s_{k}^{ \pm} \equiv s_{k}^{x} \pm i s_{k}^{y}$. The set-index $I$ will thus generally incorporate the indices for a set of lattice sites. We discuss particular choices of $\left\{|\Phi\rangle, C_{I}^{+}\right\}$in more detail below in the context of a specific example.

The exact ground-state energy eigenket and eigenbra vectors, $|\Psi\rangle$ and $\langle\widetilde{\Psi}|$ respectively, of a many-body system described by a Hamiltonian $H$, satisfy the Schrodinger equations,

$$
H|\Psi\rangle=E_{\mathrm{g}}|\Psi\rangle ; \quad\left\langle\left.\tilde{\Psi}\right|_{H}=E_{\mathrm{g}}\langle\tilde{\Psi}| .\right.
$$

We have introduced the tilde notation on the bra state, $\langle\widetilde{\Psi}|$, to remind ourselves of the fact that although the exact $\langle\widetilde{\Psi}|$ is self-evidently related to $|\Psi\rangle$ by Hermiticity, the CCM parametrizations of $|\Psi\rangle$ and $\langle\widetilde{\Psi}|$ do not manifestly preserve this Hermiticity as explained below. 
Furthermore, the tilde notation also serves to recall that even if the exact Hermiticity holds, $\langle\widetilde{\Psi}|$ differs from $(|\Psi\rangle)^{\dagger}$ by a normalization constant, as discussed below.

The ket and bra states are now specified within the single-reference normal CCM as,

$$
\begin{array}{ll}
|\Psi\rangle=\exp (S)|\Phi\rangle ; & S=\sum_{I \neq 0} S_{I} C_{I}^{+}, \\
\langle\tilde{\Psi}|=\langle\Phi| \tilde{S} \exp (-S) ; & \widetilde{S}=1+\sum_{I \neq 0} \tilde{S}_{I} C_{I}^{-},
\end{array}
$$

where the tilde notation on the operator $\widetilde{S}$ is not intended to convey any direct mathematical relationship between $\widetilde{s}$ and $s$. Rather, equation (5) defines the operators $S$ and $\widetilde{S}$. We note that the correlation operator $S$ is decomposed entirely in terms of the multiconfigurational creation operators $\left\{C_{I}^{+}\right\}$, and similarly for $\widetilde{S}$ in terms of the destruction operators $\left\{C_{I}^{-}\right\}$. We further note that although the manifest Hermiticity, $(\langle\widetilde{\Psi}|)^{\dagger}=$ $|\Psi\rangle\langle\langle\Psi \mid \Psi\rangle$, is lost, the intermediate normalization condition, $\langle\widetilde{\Psi} \mid \Psi\rangle=\langle\Phi \mid \Psi\rangle=\langle\Phi \mid \Phi\rangle \equiv 1$ is imposed. Furthermore, the correlation coefficients $\left\{s_{I}, \widetilde{s}_{I}\right\}$ are regarded as being independent variables, even though formally we have the relation,

$$
\langle\Phi| \tilde{S}=\frac{\langle\Phi| \exp \left(s^{\dagger}\right) \exp (s)}{\left\langle\Phi\left|\exp \left(s^{\dagger}\right) \exp (s)\right| \Phi\right\rangle} .
$$

In particular, the full set of coefficients $\left\{s_{I}, \tilde{s}_{I}\right\}$ provides a complete CCM description of the many-body ground state. For example, an arbitrary operator $A$ has a ground-state expectation value,

$$
\begin{aligned}
\bar{A} & \equiv\langle\tilde{\Psi}|A| \Psi\rangle=\left\langle\Phi\left|\tilde{S} \exp (-S)_{A} \exp (S)\right| \Phi\right\rangle \\
& =\bar{A}\left(\left\{S_{I}, \tilde{S}_{I}\right\}\right) .
\end{aligned}
$$

We note that the specific parametrization of equation (5) for $\langle\widetilde{\Psi}|$ is consistent with the Hellmann-Feynman theorem [5] even though it does not manifestly preserve the Hermiticity relation with $|\Psi\rangle$. Furthermore, and very importantly, this parametrization for $\langle\widetilde{\Psi}|$ is actually derivable from the Hellmann-Feynman theorem if one chooses the specific functional form for the energy, $E_{\mathrm{g}}=\langle\Phi|\exp (-S) H \exp (S)| \Phi\rangle$, which immediately follows from equations (4) and (5).

As is by now well known, the exponentiated form of the ground-state eigenket parametrization of equation (5) ensures both the proper counting of the independent fluctuations of excited multi-spin configurations (described by the set-index $I$ ) with respect to $|\Phi\rangle$, which are present in the exact ground state $|\Psi\rangle$, and the exact incorporation of the linked cluster theorem of Goldstone. The latter, in turn, guarantees the sizeextensivity of all relevant physical quantities, and thus allows us to work in the CCM directly in the thermo- dynamic limit, $N \rightarrow \infty$, where $N$ is the number of lattice spins.

By taking appropriate projections of the ground-state bra and ket Schrodinger equations (i.e. with states $C_{I}^{+}|\Phi\rangle$ and $\langle\Phi| C_{I}^{-}$, respectively), we obtain coupled sets of equations which may be solved to obtain the coefficients $\left\{s_{I}\right\}$ and $\left\{\tilde{s}_{I}\right\}$. Completely equivalently, the correlation coefficients $\left\{s_{I}, \widehat{S}_{I}\right\}$ may be determined variationally by requiring that the ground-state energy functional $H\left(\left\{s_{I}, S_{I}\right\}\right)$, defined as in equation (7), is stationary with respect to variations in each of the (independent) variables of the full set. The following coupled sets of equations are thereby easily derived,

$$
\begin{aligned}
& \delta \bar{H} / \delta \tilde{S}_{I}\left.=0 \Rightarrow \chi \Phi\left|C_{I}^{-} \exp (-s) H \exp (s)\right| \Phi\right\rangle=0, \\
& \forall I \neq 0 ; \\
&\left.\delta \bar{H} / \delta S_{I}=0 \Rightarrow \chi \Phi\left|\tilde{S} \exp (-s)\left[H, C_{I}^{+}\right] \exp (s)\right| \Phi\right\rangle=0,
\end{aligned}
$$

Equation (8) also ensures that the ground-state energy at the stationary point has the simple form

$$
E_{\mathrm{g}}=E_{\mathrm{g}}\left(\left\{S_{I}\right\}\right)=\langle\Phi|\exp (-s) H \exp (s)| \Phi\rangle,
$$

which also follows simply by projecting the ground-state ket equation (4) with $\langle\Phi| \exp (-S)$. We note that this bivariational formulation does not, however, lead to an upper bound for $E_{\mathrm{g}}$ when the summations over configurations $\{I\}$ in equation (5) for $S$ and $\widetilde{S}$ are truncated in specific approximations, since the exact Hermiticity between $|\Psi\rangle$ and $\langle\widetilde{\Psi}|$ will thereby be lost. On the other hand, it is important to note that the HellmannFeynman theorem is preserved in all such approximations, and for many purposes this may be of greater usefulness than the variational bound on the energy.

Equation (8) clearly represents a coupled set of nonlinear multinomial equations for the $c$-number correlation coefficients $\left\{S_{I}\right\}$. The well-known nested commutator expansion for the similarity-transformed Hamiltonian,

$$
\begin{aligned}
\hat{H} & \equiv \exp (-S) H \exp (s) \\
& \left.=H+[H, S]+\frac{1}{2 !}[H, S] S\right]+\cdots
\end{aligned}
$$

taken together with the fact that all of the individual components of $s$ in the sum in equation (5) commute with one another, imply that each element of $S$ in equation (5) is thus directly linked to the Hamiltonian in each of the non-vanishing terms in equation (11). Each of the coupled set of equations (8) is hence of linked cluster type. What is more, each of these equations is also of finite length when expanded using equation (11), since this otherwise infinite series will actually terminate at a finite order here, provided only that each term in the Hamiltonian $H$ contains a finite number of single-site 
spin operators, as is usually the case. The CCM parametrization thus leads in a very natural way to a workable scheme, which can also be efficiently implemented as described in more detail below. It is also important to realize that the similarity transformation lies at the heart of the CCM and is one of its most vital ingredients. It may be contrasted with its unitary transformation counterpart in a standard variational formulation in which the bra state $\langle\widetilde{\Psi}|$ is manifestly taken as being proportional to the Hermitian adjoint of $|\Psi\rangle$, as in equation (6).

We turn now to the choice of $|\Phi\rangle$ and the operators $\left\{C_{I}^{+}\right\}$for spin-lattice problems. To be specific we restrict ourselves henceforth to spin- $\frac{1}{2}$ quantum antiferromagnets in regions where the corresponding classical limit is described by a generalized Néel-like ordering in which all spins on each sublattice are separately aligned in the coordinates of a global spin quantization axis and corresponding global spin axes. In such cases it is a simple matter (and see section 3 for specific details in the case considered here) to introduce a different local quantization axis and spin coordinates on each sublattice, by a suitable rotation in spin space, so that the corresponding Néel-like state becomes a fully aligned ('ferromagnetic') state in the local axes. This 'ferromagnetic' state is chosen as the uncorrelated CCM model state, $|\Phi\rangle$, in which all spins point, say, along the respective negative $z$ axis of the corresponding local frames,

$$
|\Phi\rangle=\bigotimes_{i=1}^{N}|\downarrow\rangle_{i} \text {; in the local quantization axes. }
$$

Thus, in the local spin coordinates,

$$
s_{k}^{z}|\Phi\rangle=-\frac{1}{2}|\Phi\rangle
$$

for any arbitrary site $k$.

The correlation operator $s$ of equation (5) may now be decomposed wholly in terms of sums of products of single spin-raising operators, $s_{k}^{+}$, again defined with respect to the local quantization axes. Thus, we may write,

$$
\begin{aligned}
S & =\sum_{i_{1}} S_{i_{1}} s_{i_{1}}^{+}+\sum_{i_{1}, i_{2}} S_{i_{1} i_{2}} s_{i_{1}}^{+} s_{i_{2}}^{+}+\cdots \\
& =\sum_{n=1}^{+} \sum_{i_{1} \cdots i_{n}}^{+} S_{i_{1} \cdots i_{n}} s_{i_{1}}^{+} \ldots s_{i_{n}}^{+},
\end{aligned}
$$

where $S_{i_{1} i_{2} \cdots i_{n}}$ are the corresponding (symmetric) spincorrelation coefficients specified by the sets of site indices $\left\{i_{1}, i_{2}, \cdots, i_{n}\right\}$ on the regular lattice under consideration. The corresponding expansion for the operator $\widetilde{S}$ of equation (5) is given by

$$
\widetilde{S}=1+\sum_{n=1}^{N} \sum_{i_{1} \cdots i_{n}} \widetilde{S}_{i_{1} \cdots i_{n}} s_{i_{1}}^{-} \cdots s_{i_{n}}^{-} .
$$

The coefficients $\left\{S_{i_{1} \cdots i_{n}}, \widetilde{S}_{i_{1} \cdots i_{n}} ; n=1,2, \ldots, N\right\}$ are thus what we denoted generically by the set $\left\{s_{I}, \tilde{s}_{I}\right\}$ previously. Equation (8) thus yields the specific set of coupled nonlinear CCM equations,

$$
\left\langle\Phi\left|s_{i_{1}} s_{i_{2}}^{-} \cdots s_{i_{n}}^{-} \exp (-s) H \exp (s)\right| \Phi\right\rangle=0,
$$

to determine the correlation coefficients $\left\{S_{i_{1} i_{2} \cdots i_{n}}\right\}$.

We note that we may map the sets of coefficients $\left\{S_{i_{1} \cdots i_{n}}\right\}$ and $\left\{\tilde{S}_{i_{1} \cdots i_{n}}\right\}$ onto sets $\left\{\chi_{r}\right\}$ and $\left\{\tilde{\chi}_{r}\right\}$ respectively, where $r$ labels the independent or fundamental configurations, i.e. only those that are inequivalent under the lattice symmetries (namely, translations, rotations, and reflections) of the Hamiltonian and under permutations of the indices. The sets $\left\{x_{r}\right\}$ and $\left\{\tilde{x}_{r}\right\}$ are thus defined to count the independent correlation coefficients associated with each fundamental configuration once and once only. Generally speaking there will be $N v_{r}\left(n_{r}\right)$ ! equivalent configurations on the lattice associated with each fundamental configuration, where $n_{r}$ is the number of sites in the $r$ th configuration and $\left(n_{r}\right)$ ! is the combinatorial factor associated with permutations of the $n_{r}$ indices, the factor $N$ arises from the translations, and the factor $v_{r}$ is the replication factor of the $r$ th configuration associated with the point symmetry group (or sub-group) of transformations on the lattice which preserve the Hamiltonian. In particular, we need only to consider one of the $N v_{r}\left(n_{r}\right)$ ! equivalent sets of equations (15) associated with each independent coefficient $\chi_{r}$.

At this point we need to consider approximation schemes whereby the expansions of $S$ and $S$ in equations $(14 a)$ and $(14 b)$ may be truncated to some finite or infinite subset of the full set of independent (fundamental) multi-spin configurations. The three most commonly employed schemes have been: (1) the SUB $n$ scheme, in which all correlations involving only $n$ or fewer spins are retained, but no further restriction is made concerning their spatial separation on the lattice; (2) the $\mathrm{SUB}_{n-m}$ sub-approximation, in which all $\mathrm{SUB} n$ correlations spanning a range of no more than $m$ adjacent lattice sites are retained; and (3) the localized LSUB $m$ scheme, which retains all multi-spin correlations over all possible distinct locales on the lattice defined by $m$ or fewer contiguous sites. For the results reported below we adopt only the LSUB $m$ scheme here.

The practical implementation of the CCM thus now consists of first enumerating all distinct multi-spin correlation configurations retained in the selected approximation, and then generating the corresponding set of CCM equations. The first stage is essentially a problem in graph theory, whereas equation (15) shows that the second stage involves two distinct computational aspects 
in the calculation of all possible non-zero contributions to the matrix element on the left-hand side of this equation. Thus, the first step is to calculate the similaritytransformed Hamiltonian, $H=\exp (-S) H \exp (S)$, and hence $\hat{H}|\Phi\rangle$; while the second is to select those terms of $\hat{H}|\Phi\rangle$ which match exactly the string of spin-lowering operators represented by the set of site indices $\left\{i_{1}, i_{2}, \ldots, i_{n}\right\}$, and which hence give a non-zero overlap. Clearly, the former problem is intrinsically related to the non-commutative nature of quantum spin operators, and relies only on the algebraic relations of equation (3); while the latter problem is intrinsically related to the geometric properties of the spatial lattice under consideration, and is essentially a problem of patternmatching. We have shown elsewhere [29] how each of the above stages may very efficiently be implemented computationally to very high orders, to yield a set of coupled CCM ket-state equations which may then be solved by standard Newton-Raphson techniques. The bra-state equations are also handled in an analogous fashion.

In the following section the power of the CCM is illustrated by application to the spin $-\frac{1}{2}$ anisotropic Heisenberg (or $X X Z$ ) model on an infinite 2D square lattice.

\section{The spin- $-\frac{1}{2} X X Z$ antiferromagnet on the $2 D$ square lattice \\ 3.1. The Hamiltonian}

The $X X Z$ Hamiltonian is specified in terms of global spin coordinates as follows,

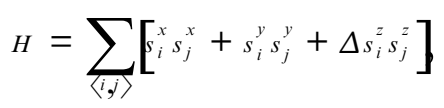

where the sum on $\langle i, j\rangle$ runs over all nearest-neighbour pairs and counts each pair once only. We note that on the $2 \mathrm{D}$ square lattice this model has no exact solution, unlike its $1 \mathrm{D}$ chain counterpart which has been shown to be exactly integrable (and hence solved) using the Bethe ansatz. The $X X Z$ model appears to have at least three distinct regimes: an Ising-like phase for sufficiently large values of the anisotropy parameter $\Delta$, which is characterized by non-zero Néel order wherein nearestneighbour spins in the ground-state wave function align antiparallel along the $z$ axis; a planar-like phase in which the spins in the ground-state wave function are believed to lie in the $x y$ plane; and a ferromagnetic phase.

Barnes and his co-workers [30] have performed a Monte Carlo study of the 2D $X X Z$ model on a square lattice. They observed that while the staggered magnetization is non-zero in the $z$ direction for $\Delta>1$, it appears to fall to zero below $\Delta=1$. They conclude that there is a phase transition at or very near to the Heisenberg point $\Delta=1$, exactly as in the $1 \mathrm{D}$ case. Kubo and Kishi [31] have also investigated the ground state of the $2 \mathrm{D}$ square lattice $X X Z$ model by making use of exact sum rules. They found that the ground state possesses an off-diagonal long-range order similar to that of the $X Y$ model at small values of the anisotropy parameter, $0.0<$ $\Delta<0.13$. They also found that for values $\Delta>1.78$ the system possesses non-zero Ising-like long-range order. Finally, at $\Delta=-1$ there is a first-order phase transition to a ferromagnetic phase which exists for all $\Delta<-1$ for this model. Although there are no exact proofs available, it is widely believed that the model has a phase (or perhaps more than one phase) with planar-like order for $-1<\Delta<1$, and an Ising-like phase with Néel-like antiferromagnetic order along the $z$ axis for $\Delta>1$.

The isotropic Heisenberg point $(\Delta=1)$ is thus expected to be a critical point marking the transition between states of different quantum order. We shall use it here as a specific example to test the ability of the CCM to predict phase transitions and its potential to discuss the critical phenomena (e.g. the critical indices) at this point. For the sake of later comparisons we note that Runge [32] has performed the most accurate Monte Carlo simulations available up until now for the square-lattice spin- $\frac{1}{2}$ isotropic Heisenberg antiferromagnet. By performing simulations on lattices up to size $16 \times 16$, and by extrapolating to the infinite lattice limit, $N \rightarrow \infty$, he finds a value for the ground-state energy per spin of $E_{\mathrm{g}} / N=-0.66934 \pm 0.00004$, and a value for the sublattice magnetization, $\mathrm{M}^{+}$, which is $(61.5 \pm 0.5) \%$ of the classical value. By comparison, linear spin-wave theory (LSWT) [33] gives a value of $E_{\mathrm{g}} / N=-0.658$ and a value for $M^{+}$which is $60.6 \%$ of the classical value.

\subsection{Choice of CCM model state}

There is never a unique choice of model state $|\Phi\rangle$. Our choice should usually be guided by any physical insight available to us concerning the system or, more specifically, that particular phase of it which is under consideration. In the absence of any insight into the quantum many-body system one may sometimes be guided by the behaviour of the corresponding classical system. The $X X Z$ model under consideration provides just such an illustrative example. Thus, for $\Delta>1$ the classical Hamiltonian of equation (16) on the 2D square lattice (and, indeed, on any bipartite lattice) is minimized by a perfectly antiferromagnetically Néel-ordered state in the $z$ direction, whereas for $-1<\Delta<1$ it is minimized by a correspondingly ordered state with spins antiferromagnetically aligned along any direction in the $x y$ plane, say along the $x$ axis. Thus, we see that even for the same spin model and lattice, different choices of model state may be preferable, depending 
on the particular regime of parameter space in which we are interested. For present purposes we shall utilize both of these classical Néel states, namely the $z$-aligned Néel state and the $x$-aligned Néel state (with the latter henceforth denoted as the planar model state), for two separate sets of corresponding CCM calculations. In both cases we now set up different local sets of spin axes on both sublattices so that in the local coordinates all spins in both model states point in the negative $z$ direction, as discussed above in section 2 (and see equation (12)).

For the $z$-aligned Néel state we simply perform a rotation of the axes of the up-pointing spins by $180^{\circ}$ about the $y$ axis, such that

$$
s^{x} \rightarrow-s^{x}, \quad s^{y} \rightarrow s^{y}, \quad s^{z} \rightarrow-s^{z} .
$$

The Hamiltonian of equation (16) may now be written in these local axes as

$$
H^{z}=-\frac{1}{2} \sum_{\langle i, j\rangle}\left[s_{i}^{+} s_{j}^{+}+s_{i}^{-} s_{j}^{-}+2 \Delta s_{i}^{z} s_{j}^{z}\right]
$$

where the superscript $z$ on $H^{z}$ reminds us that the Hamiltonian is written in the local spin coordinate axes appropriate to the $z$-aligned Néel model state.

In order to produce a 'ferromagnetic' model state, as in equation (12), for the planar model state in the local frames, we rotate the axes of the left-pointing spins (i.e. those pointing in the negative $x$ direction) in the planar state by $90^{\circ}$ about the $y$ axis, and the axes of the corresponding right-pointing spins by $-90^{\circ}$ about the $y$ axis. (Note that the positive $z$ axis is defined here to point upwards and the positive $x$ axis is defined to point rightwards.) Thus, the transformations of the local axes are described such that

$$
s^{x} \rightarrow s^{z}, \quad s^{y} \rightarrow s^{y}, \quad s^{z} \rightarrow-s^{x}
$$

for the left-pointing spins, and such that

$$
s^{x} \rightarrow-s^{z}, \quad s^{y} \rightarrow s^{y}, \quad s^{z} \rightarrow s^{x}
$$

for the right-pointing spins. The transformed Hamiltonian of equation (16) may now be written in these local axes as

$$
\begin{aligned}
H^{\mathrm{p}}= & -\frac{1}{4} \sum_{\langle i, j\rangle}\left[(\Delta+1)\left(s_{i}^{+} s_{j}^{+}+s_{i}^{-} s_{j}^{-}\right)\right. \\
& \left.+(\Delta-1)\left(s_{i}^{+} s_{j}^{-}+s_{i}^{-} s_{j}^{+}\right)+4 s_{i}^{z} s_{j}^{z}\right]
\end{aligned}
$$

where, again, the superscript $\mathrm{p}$ on ${ }^{\mathrm{p}}$ reminds us that the Hamiltonian is written in the local spin coordinate axes appropriate to the planar model state. It is important to recall that since the Hamiltonians $H, H^{z}$, and $H^{\mathrm{p}}$ of equations (16), (18), and (21) differ only by similarity transformations their eigenvalue spectra are identical.

\subsection{Enumeration of the independent correlation configurations}

The first step in the practical implementation of the $\mathrm{CCM}$ at the $\mathrm{LSUB} m$ level of approximation discussed in section 2 is to enumerate all of the distinct multi-spin configurations or correlated clusters, which we shall henceforth call fundamental configurations, $\left\{i_{1}, i_{2}, \ldots, i_{n}\right\}$ with $n<_{m}$, which are retained at the LSUB $m$ level. Only those configurations which cannot be obtained from one another using the lattice symmetries (namely, translations, rotations and reflections) shared by the Hamiltonian are to be counted as distinct. For the square lattice under consideration there are four rotational operations, $\left(0^{\circ}, 90^{\circ}, 180^{\circ}, 270^{\circ}\right)$, and four reflections (along the $x$ and $y$ axes, and along the lines $y= \pm x$ ), which preserve the symmetries of both the lattice and the Hamiltonian. Each such fundamental configuration is associated with two single independent correlation coefficients $\chi_{r}$ and $\tilde{x}_{r}$ associated with the cluster operators $S$ and $\tilde{S}$ respectively, as discussed in section 2. Each correlated cluster may be either a connected cluster of size $m$ (also known as a 'lattice animal' or 'polyomino') or a connected or disconnected subset of it. We note that the enumeration of the number of lattice animals of size $m$ on a regular lattice as $m$ becomes large remains an open problem in combinatorial graph theory. However, efficient algorithms for their enumeration for $m \leq 20$ have been developed in such fields as cell growth and percolation theory.

We also note that although $H^{\mathrm{p}}$ and $H^{z}$ are fully equivalent to one another, the number of fundamental LSUB $m$ configurations at each level $m>2$ is greater for $H^{\mathrm{p}}$ than for $H^{z}$ due to other constraints which arise from symmetries of the Hamiltonian. Thus, firstly, we note that both $H^{\mathrm{p}}$ and $H^{z}$ contain only even products of spin-flip operators (plus a single term containing two $s^{z}$ operators). Repeated application of either Hamiltonian to the model state $|\Phi\rangle$ will thus only create states with an even number of spin flips with respect to it. Since the exact ground state may be obtained as a linear combination of states obtained by repeated application of the Hamiltonian to $|\Phi\rangle$, assuming only that $|\Phi\rangle$ is not orthogonal to the exact ground state, we may hence restrict ourselves to LSUB $m$ fundamental configurations which contain an even number of spin flips, i.e. to coefficients $S_{i_{1} i_{2} \cdots i_{n}}$ where $n$ is even.

Secondly, we note that the number of fundamental configurations can be further reduced for $H^{z}$. Thus, $H^{z}$ has the additional feature that when applied to $|\Phi\rangle$ it produces states with the same number of spin flips on both sublattices, and hence we can further restrict the fundamental configurations to those which preserve this feature. This symmetry is related to the fact that the original Hamiltonian, and hence also $H^{\mathrm{p}}$ and $H^{z}$, com- 
mute with the $z$ component of the total uniform magnetization, $s_{T}^{z}=\sum_{i} s_{i}^{z}$ (where $s_{i}^{z}$ is defined with respect to the original global quantization axis, and the sum over the index $i$ runs over all $N \rightarrow \infty$ lattice sites). However, whereas the $z$-aligned Néel model state is an eigenstate of $s_{T}^{z}$, the planar model state is not. Hence, for the $z$ aligned Néel model state one may explicitly conserve $s_{T}^{z}$ (as zero for the antiferromagnetic sector) by restricting the fundamental configurations to those which produce no change in $s_{T}^{z}(=0)$. We note the number of fundamental configurations up to the LSUB8 level of approximation for both model states in table 1 . The actual calculations reported below are performed up to this level for $H^{z}$, but only up to LSUB6 level for $H^{\mathrm{p}}$ due to the increased number of fundamental configurations in the latter case.

We note also that $H^{\mathrm{p}}$ and $H^{z}$ become identical at the Heisenberg point $\Delta=1$. In the actual calculations this is reflected by the fact that the additional cluster correlation coefficients at a given LSUB $m$ level included for $H^{\mathrm{p}}$ beyond those included for $H^{z}$ become zero at this point, $\Delta \rightarrow 1^{-}$. Further details of the enumeration of the independent configurations is contained in [29]

\subsection{The CCM ket-state equations}

In order to evaluate the CCM ket-state equations (15), we first require the similarity-transformed Hamiltonians, $\hat{H}^{z}$ and $\hat{H}^{\mathrm{p}}$, defined as in equation (11). By making use of the fundamental $S U(2)$ commutation relations of equation (3) it is straightforward to show that after letting $\hat{H}^{z}$ act on $|\Phi\rangle$, as needed in equation (15), we have

$$
\hat{H}^{z}|\Phi\rangle \equiv \exp (-S) H^{z} \exp (S)|\Phi\rangle=\left(\hat{H}_{1}^{z}+\hat{H}_{2}^{z}+\hat{H}_{3}^{z}\right)|\Phi\rangle
$$

where

$$
\begin{aligned}
\hat{H}_{1}^{z}= & -\frac{1}{2} \Delta \sum_{k \rho}\left(F_{k}^{z} F_{m}^{z}+G_{k m}^{z}\right) s_{k}^{+} s_{m}^{+} \\
& -\frac{1}{4} \sum_{k \rho}\left[1+\left(F_{k}^{z}\right)^{2}\left(F_{m}^{z}\right)^{2}+4 F_{k}^{z} F_{m}^{z} G_{k m}^{z}\right. \\
& \left.+2\left(G_{k m}^{z}\right)^{2}\right]_{k}^{+} s_{m}^{+}, \\
\hat{H}_{2}^{z}= & \frac{1}{4} \Delta \sum_{k \rho}\left(F_{k}^{z} s_{k}^{+}+F_{m}^{z} s_{m}^{+}\right) \\
& +\frac{1}{4} \sum_{k \rho}\left(F_{k}^{z} F_{m}^{z}+2 G_{k m}^{z}\right)\left(F_{k}^{z} s_{k}^{+}+F_{m}^{z} s_{m}^{+}\right), \\
\hat{H}_{3}^{z}= & -\frac{1}{8} \sum_{k \rho}\left[\Delta+2\left(F_{k}^{z} F_{m}^{z}+G_{k m}^{z}\right)\right]
\end{aligned}
$$

and where $k$ runs over all lattice sites, and $m \equiv k+\rho$ such that $\rho$ runs over all 4 nearest neighbours to $k$ on the lattice. The operators $F_{k}$ and $G_{k m}$ are defined generically as follows,

$$
\begin{aligned}
F_{k} & \equiv \sum_{l=1}^{\infty} l \sum_{i_{1} \cdots i_{l-1}} s_{k i_{1} \cdots i_{l-1}} s_{i_{1}}^{+} \cdots s_{i_{l-1}}^{+}, \\
G_{k m} & \equiv \sum_{l=2}^{\infty} l(l-1) \sum_{i_{1} \cdots i_{l-2}} s_{k m i_{1} \cdots i_{l-2}} s_{i_{1}}^{+} \cdots s_{i_{l-2}}^{+},
\end{aligned}
$$

and $F_{k}^{z}$ and $G_{k m}^{z}$ are the particular cases where $S_{i_{1} \cdots i_{n}} \rightarrow$ $S_{i_{1} \cdots i_{n}}^{z}$, namely the cluster correlation coefficients obtained by use of the present $z$-aligned Néel state and $H^{2}$. Equation (25) shows that the ground-state energy for the $z$-aligned Néel model state is simply given in terms of $x_{1}^{z} \equiv S_{k, k+\rho}^{z}$, the nearest-neighbour two-body cluster correlation coefficient obtained by using $H^{z}$ and the $z$-aligned Néel model state, as

$$
\frac{E_{\mathrm{g}}}{N}=-\frac{1}{2}\left(4 x_{1}^{z}+\Delta\right) \text {. }
$$

We note that $x_{1}^{z} \equiv S_{k, k+\rho}^{z}$ is independent of $k$ and $\rho$ by the translational and rotational symmetries of the lattice.

The equivalent relations for $\hat{H}^{\mathrm{p}}$ are obtained as follows,

$$
\hat{H}^{\mathrm{p}}|\Phi\rangle \equiv \exp (-S)_{H}^{\mathrm{p}} \exp (S)|\Phi\rangle=\left(\hat{H}_{1}^{\mathrm{p}}+\hat{H}_{2}^{\mathrm{p}}+\hat{H}_{3}^{\mathrm{p}}\right)|\Phi\rangle,
$$

where

$$
\begin{aligned}
& \hat{H}_{1}^{\mathrm{p}}=\frac{1}{2} \sum_{k \rho}\left\{-\left(F_{k}^{\mathrm{p}} F_{m}^{\mathrm{p}}+G_{k m}^{\mathrm{p}}\right)+\frac{1}{4}(\Delta-1)\right. \\
& \left.\times\left[\left(F_{k}^{\mathrm{p}}\right)^{2}+\left(F_{m}^{\mathrm{p}}\right)^{2}\right]\right\} s_{k}^{+} s_{m}^{+} \\
& -\frac{1}{8}(\Delta+1) \sum_{k \rho}\left[1+\left(F_{k}^{\mathrm{p}}\right)^{2}\left(F_{m}^{\mathrm{p}}\right)^{2}+4 F_{k}^{\mathrm{p}} F_{m}^{\mathrm{p}} G_{k m}^{\mathrm{p}}\right. \\
& \left.+2\left(G_{k m}^{\mathrm{p}}\right)^{2}\right]_{k}^{+} s_{m}^{+}
\end{aligned}
$$

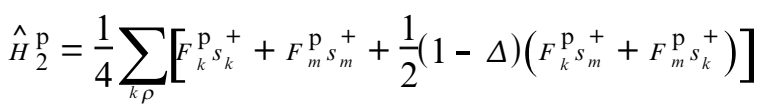

$$
\begin{aligned}
& +\frac{1}{8}(\Delta+1) \sum_{k \rho}\left(2 G_{k m}^{\mathrm{p}}+F_{k}^{\mathrm{p}} F_{m}^{\mathrm{p}}\right)\left(F_{k}^{\mathrm{p} s_{k}^{+}}+F_{m}^{\mathrm{p}} s_{m}^{+}\right), \\
& \hat{H}_{3}^{\mathrm{p}}=-\frac{1}{8} \sum_{k \rho}\left[1+(\Delta+1)\left(F_{k}^{\mathrm{p}} F_{m}^{\mathrm{p}}+G_{k m}^{\mathrm{p}}\right)\right]
\end{aligned}
$$

The operators $F_{k}^{\mathrm{p}}$ and $G_{k m}^{\mathrm{p}}$ are again defined as in equations (26) and (27), but with the cluster correlation coefficients $S_{i_{1} \cdots i_{n}} \rightarrow S_{i_{1} \cdots i_{n}}^{\mathrm{p}}$ obtained by use of the present 
planar model state and $H^{\mathrm{p}}$. Equation (32) immediately yields,

$$
\frac{E_{\mathrm{g}}}{N}=-\frac{1}{2}\left[2 x_{1}^{\mathrm{p}}(\Delta+1)+1\right]
$$

where $x_{1}^{\mathrm{p}} \equiv S_{k, k+\rho}^{\mathrm{p}}$, the nearest-neighbour two-body cluster correlation coefficient obtained by using $H^{\mathrm{p}}$ and the planar model state.

In both cases the corresponding LSUB $m$ equations are obtained by evaluating all non-zero contributions to equation (15). There is one such (coupled nonlinear) equation for every fundamental configuration retained, and hence $N_{\mathrm{F}}$ equations in $N_{\mathrm{F}}$ coefficients, for each level of approximation. The actual derivation of the ket-state equations from equation (15) and equations (22)-(25) or equations (29)-(32) is thus seen to reduce essentially to a pattern-matching exercise, and we describe elsewhere [29] its efficient computational implementation. The bra-state equations can also be derived in an analogous fashion.

\section{Results \\ 4.1. Ground-state energy}

Results for the ground-state energy using the two model states are illustrated in figure 1 at the LSUB4 and LSUB6 levels of approximation, where they are compared with the Monte Carlo results of Barnes et al. [30] The highest approximation that we have undertaken for the planar model state case is LSUB6, which contains 131 independent cluster configuration coefficients and which yields an energy per spin, $E_{\mathrm{g}} / N=$ - 0.66700 at the Heisenberg point $(\Delta=1)$. Due to the reduced number of independent configurations for the case of the $z$-aligned Néel model state we have also been

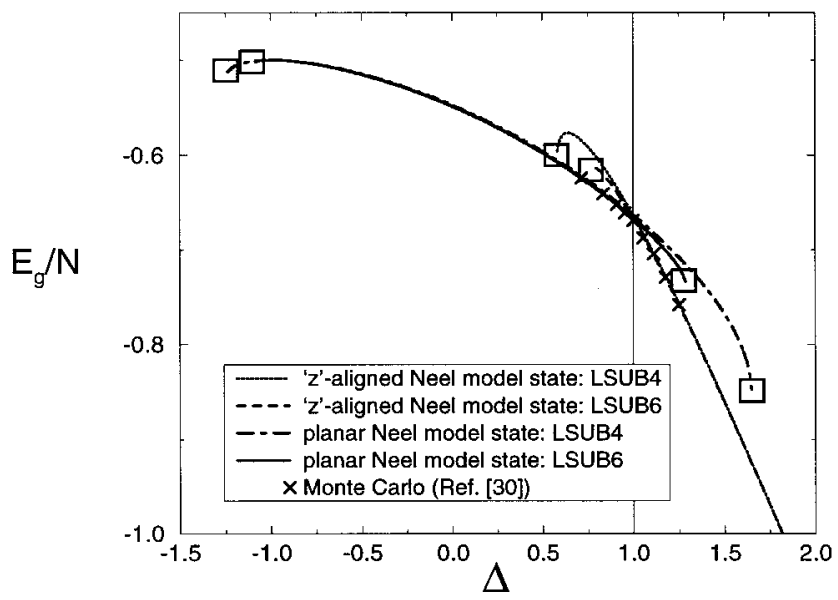

Figure 1. Results for the CCM ground-state energy of the spin- $\frac{1}{2} X X Z$ model on the $2 \mathrm{D}$ square lattice, using the LSUB $m$ approximation with $m=4,6$ based on both the planar and $z$-aligned Néel model states, compared to the Monte Carlo results of [30] LSUB $m$ critical points, $\Delta_{\mathrm{F}}^{\mathrm{p}}, \Delta_{\mathrm{A}}^{\mathrm{p}}$ and $\Delta_{\mathrm{A}}^{z}$, are indicated by the boxes. able to solve the higher LSUB8 approximation in this case. The 1287 coupled equations in this case yield a value of the energy per spin, $E_{\mathrm{g}} / N=-0.66817$ at $\Delta=1$. The results for the Heisenberg model (which are identical using both model states for this case) are summarized in table 1 , and ground-state energies are also shown in table 2 for a range of values of $\Delta$, and for calculations using both model states as CCM reference states.

In order to compare our results with those cited in section 3.1 from other methods, we attempt a simple heuristic extrapolation of our $\mathrm{LSUB} m$ results to the limit $m \rightarrow \infty$ at the isotropic Heisenberg point $(\Delta=1)$. As has already been found elsewhere [11] our results seem to extrapolate well to their asymptotic value with a leading $m$-dependent correction that scales as $m^{-2}$. In this way we obtain an extrapolated value for the ground-state energy per spin of $E_{\mathrm{g}} / N=-0.66968$. This compares very well with the best Monte Carlo simulation value [32] of $-0.66934 \pm 0.00004$, and is very much more accurate, by comparison with this Monte Carlo value, than with the linear spin-wave theory (LSWT) result [33] of $E_{\mathrm{g}} / N=-0.658$.

Figure 1 and table 2 illustrate that at a given LSUB $m$ level of approximation the CCM result for the groundstate energy using the $z$-aligned Néel model state lies lower than its counterpart using the planar model state for $\Delta>1$, and vice versa for $\Delta<1$. This result is precisely as would be expected classically, as discussed above in section 3.2, and it illustrates the power of being able to employ different CCM model states which are specifically geared to different possible phases. If we were simply to take that solution with the lower energy for each value of $\Delta$ (for which we note, however, that there is no real justification), we would infer that there is a phase transition at $\Delta=1$ between a phase with Ising-like order at $\Delta>1$ and a planar-like phase for $\Delta<1$.

We note, furthermore, and much more importantly, that each separate calculation also yields evidence of such a phase transition. Thus, we find that beyond certain critical values, $\Delta_{c}$, of the anisotropy parameter there exists no physically reasonable solution to the LSUB $m$ CCM equations for $m \geqslant 4$, as is illustrated for the cases $m=4,6$ in figure 1 . In previous work [11] we have related this characteristic breakdown of the CCM equations at certain critical points to actual phase transitions of the real system, and we explore this further in section 4.2 below. A useful means to detect phase transitions within the LSUB $m$ scheme is to calculate the so-called anisotropy susceptibility, $\chi_{\mathrm{a}}$,

$$
\chi_{\mathrm{a}} \equiv-\frac{\partial^{2}\left(E_{\mathrm{g}} / N\right)}{\partial \Delta^{2}} \text {. }
$$


Table 1. Results obtained for the $\operatorname{spin}-\frac{1}{2} \times x$ z model on the 2D square lattice using CCM LSUB $m$ approximations $(m=2,4,6,8)$. $N \mathrm{~F}$ denotes the number of fundamental configurations for the planar model state, which are further decomposed in terms of connected and disconnected ones respectively, and $N_{\mathrm{F}}^{z}$ denotes the number of fundamental configurations for the $z$ axis Néel model state. The ground-state energy per spin, $E_{\mathrm{g}} / N$, and the sublattice magnetization, $M^{+}$, at the isotropic Heisenberg point $(\Delta=1)$ are shown, as well as extrapolated results in the limit $m \rightarrow \infty$. Various critical anisotropy parameters are also given. $\Delta_{\mathrm{F}}^{\mathrm{p}}$ and $\Delta_{\mathrm{A}}^{\mathrm{p}}$ indicate the LSUB $m$ critical points for the planar model state corresponding to the ferromagnetic and antiferromagnetic phase transitions. $\Delta_{\mathrm{A}}^{z}$ indicates the critical point for the $z$-axis Néel model state corresponding to the antiferromagnetic phase transition. Note that there are no terminating points in the LSUB2 approximation.

\begin{tabular}{lccccccc}
\hline$m$ & $N_{\mathrm{F}}^{\mathrm{p}}$ & $N_{\mathrm{F}}^{z}$ & $\left.\frac{E_{\mathrm{g}}}{N}\right|_{\Delta=1}$ & $\left.M^{+}\right|_{\Delta=1}$ & $\Delta_{\mathrm{F}}^{\mathrm{p}}$ & $\Delta_{\mathrm{A}}^{\mathrm{p}}$ & $\Delta_{\mathrm{A}}^{z}$ \\
\hline 2 & $1(1+0)$ & $1(1+0)$ & -0.64833 & 0.8414 & - & - & - \\
4 & $10(6+4)$ & $7(5+2)$ & -0.66366 & 0.7648 & -1.250 & 1.648 & 0.577 \\
6 & $131(41+90)$ & $75(29+46)$ & -0.66700 & 0.7273 & -1.084 & 1.286 & 0.7631 \\
8 & $2793(410+2383)$ & $1287(259+1028)$ & -0.66817 & 0.7048 & $?$ & $?$ & 0.8429 \\
$\infty$ & - & - & -0.66968 & 0.62 & -0.95 & 1.00 & $0.96 \pm 0.04$ \\
\hline
\end{tabular}

Table 2. Results for the ground-state energy per spin of the 2D spin- $-\frac{1}{2} x$ X $Z$ model on the square lattice using both the planar and $z$-aligned Néel model states, compared with the Monte Carlo results of [30] The '—' symbol indicates values of $\Delta$ which lie outside the range, defined by the $\Delta_{\mathrm{F}}^{\mathrm{p}}, \Delta_{\mathrm{A}}^{\mathrm{p}}$, and $\Delta_{\mathrm{A}}^{z}$ critical points, in which there exists a physically reasonable solution to the LSUB $m$ CCM equations for $m \geqslant 4$. The '**' symbol indicates points at which a Monte Carlo solution has not yet been determined.

\begin{tabular}{|c|c|c|c|c|c|c|c|c|}
\hline \multirow[b]{2}{*}{$\Delta$} & \multicolumn{3}{|c|}{$\begin{array}{c}\text { CCM results based } \\
\text { on the planar Néel state }\end{array}$} & \multirow[b]{2}{*}{$\begin{array}{r}\text { Monte } \\
\text { Carlo } \\
\end{array}$} & \multicolumn{4}{|c|}{$\begin{array}{c}\text { CCM results based } \\
\text { on the } z \text {-aligned Néel state }\end{array}$} \\
\hline & LSUB2 & LSUB4 & LSUB6 & & LSUB8 & LSUB6 & LSUB4 & LSUB2 \\
\hline-1.0 & -0.5 & -0.5 & -0.5 & $* *$ & - & - & - & - 0.8483 \\
\hline-0.5 & - 0.5103 & -0.5145 & -0.5151 & $* *$ & - & - & - & - 0.5885 \\
\hline 0.0 & -0.5403 & - 0.5473 & -0.5483 & $* *$ & - & - & - & - 0.4472 \\
\hline 0.5 & - 0.5874 & - 0.5959 & -0.5975 & $* *$ & - & - & - & - 0.4885 \\
\hline 0.71429 & -0.6120 & -0.6222 & -0.6242 & -0.624 & - & - & -0.5891 & -0.5480 \\
\hline 0.83333 & -0.6267 & -0.6385 & -0.6408 & -0.641 & - & - 0.6199 & -0.6116 & -0.5875 \\
\hline 0.90909 & -0.6364 & -0.6496 & - 0.6523 & -0.652 & -0.6415 & - 0.6390 & -0.6338 & -0.6144 \\
\hline 0.95238 & -0.6420 & -0.6562 & - 0.6591 & -0.661 & -0.6535 & - 0.6518 & - 0.6477 & - 0.6304 \\
\hline 1.0 & - 0.6483 & - 0.6637 & - 0.6670 & -0.669 & -0.6682 & - 0.6670 & - 0.6637 & - 0.6483 \\
\hline 1.05263 & -0.6554 & -0.6723 & -0.6762 & -0.687 & -0.6856 & - 0.6848 & - 0.6821 & - 0.6686 \\
\hline 1.11111 & -0.6634 & -0.6823 & -0.6871 & -0.704 & -0.7062 & -0.7056 & -0.7035 & - 0.6917 \\
\hline 1.17647 & -0.6726 & - 0.6940 & -0.7005 & -0.729 & -0.7303 & - 0.7298 & - 0.7282 & -0.7180 \\
\hline 1.25 & -0.6830 & - 0.7082 & - 0.7189 & -0.759 & -0.7585 & - 0.7582 & - 0.7570 & - 0.7482 \\
\hline 1.5 & - 0.7201 & - 0.7680 & - & $* *$ & - 0.8611 & - 0.8610 & - 0.8604 & - 0.8550 \\
\hline 2.0 & -0.8000 & - & - & $* *$ & - 1.0833 & - 1.0833 & - 1.0831 & - 1.0806 \\
\hline
\end{tabular}

Since the CCM equations are known analytically, $\chi_{\mathrm{a}}$ and all other derivatives may also be calculated directly (i.e. from analytic equations). We find that $\chi_{a}$ diverges at the critical points.

More specifically, we find that for the CCM calculations based on the planar model state $\chi_{\mathrm{a}}$ diverges at critical values $\Delta_{\mathrm{c}}=\Delta_{\mathrm{F}}^{\mathrm{p}}$ and $\Delta_{\mathrm{A}}^{\mathrm{p}}$, corresponding to the ferromagnetic and antiferromagnetic phase transitions respectively, for all $\mathrm{LSUB} m$ approximations with $m>2$. These results are illustrated in table 1 , which also displays the single critical point at $\Delta_{\mathrm{c}}=\Delta_{\mathrm{A}}^{z}$ for the CCM calculations based on the $z$-aligned Néel model state, and which again corresponds to the antiferromagnetic phase transition. As one might hope, the position of the critical point $\Delta_{\mathrm{F}}^{\mathrm{p}}$ becomes closer to the true ferromagnetic phase transition at $\Delta=-1$ as the approximation level is increased. Also, both $\Delta_{\mathrm{A}}^{\mathrm{p}}$ and $\Delta_{\mathrm{A}}^{z}$ appear to converge with increasing LSUB $m$ index $m$, and for a given value of $m$ always bound the point $\Delta=1$ at which the true antiferromagnetic phase transition is believed (by symmetry arguments) to lie. We have shown elsewhere [11] how the corresponding SUB2-m 
results for $\Delta_{\mathrm{A}}^{z}$ seem to approach the full SUB2 value as $m^{-2}$, and the same rule fitted to the $\operatorname{LSUB} m$ results yields the corresponding predictions for the extrapolated antiferromagnetic point indicated in table 1 , namely $\Delta_{\mathrm{A}}^{z} \approx 0.96 \pm 0.04$, and $\Delta_{\mathrm{A}}^{\mathrm{p}} \approx 1.00$. Both predictions are compatible with each other and with the expected value $\Delta_{\mathrm{A}}=1$.

\subsection{Sublattice magnetization}

In order to discuss the phase transition further it is necessary to consider the degree of quantum order inherent in the CCM wave functions obtained at the various LSUB $m$ levels of approximation, and based on both model states. The simplest such order parameter is the sublattice magnetization, $M^{+} \equiv-2\left\langle s^{z}\right\rangle$, which is defined as the average over the entire lattice of $s^{z}$ in the local (rotated) spin coordinates, or, equivalently over a single sublattice of the corresponding unrotated component of the spin in the original global coordinates. Thus, $M^{+}=1$ for both model states, with perfect antiferromagnetic alignment along the global $z$ axis for the $z$-aligned state and along the global $x$ axis for the planar state. Quantum fluctuations (i.e. multi-spin correlations) present in the exact interacting ground state are expected to reduce $M^{+}$below unity. We would expect, a priori, that a phase transition would be marked by $M^{+}$ becoming zero (or, in an approximate calculation, singular) at some critical value of the coupling parameter, namely $\Delta$ for the present $X X Z$ model.

By inserting the CCM parametrizations of equation (5) we find,

$$
\begin{aligned}
M^{+} & =-\frac{2}{N} \sum_{k=1}^{N}\left\langle\tilde{\Psi}\left|s_{k}^{z}\right| \Psi\right\rangle \\
& =-\frac{2}{N} \sum_{k=1}^{N}\left\langle\Phi\left|\tilde{S} \exp (-S) s_{k}^{z} \exp (S)\right| \Phi\right\rangle,
\end{aligned}
$$

where $s_{k}^{z}$ is in the local coordinates of each sublattice. In the notation of equation (26) we find

$$
\begin{aligned}
M^{+} & =1-\frac{2}{N} \sum_{k=1}^{N}\left\langle\Phi\left|\tilde{S} F_{k} S_{k}^{+}\right| \Phi\right\rangle \\
& =1-\frac{2}{N} \sum_{n=1}^{\infty} n(n !) \sum_{i_{1} \cdots i_{n}} \widetilde{S}_{i_{1} \cdots i_{n}} S_{i_{1} \cdots i_{n}}
\end{aligned}
$$

The sum in equation (36) may be rewritten in terms of the independent correlation coefficients $\tilde{\chi}_{r}$ and $x_{r}$ associated with the $N_{\mathrm{F}}$ fundamental configurations of a given $\operatorname{LSUB} m$ approximation, which were introduced

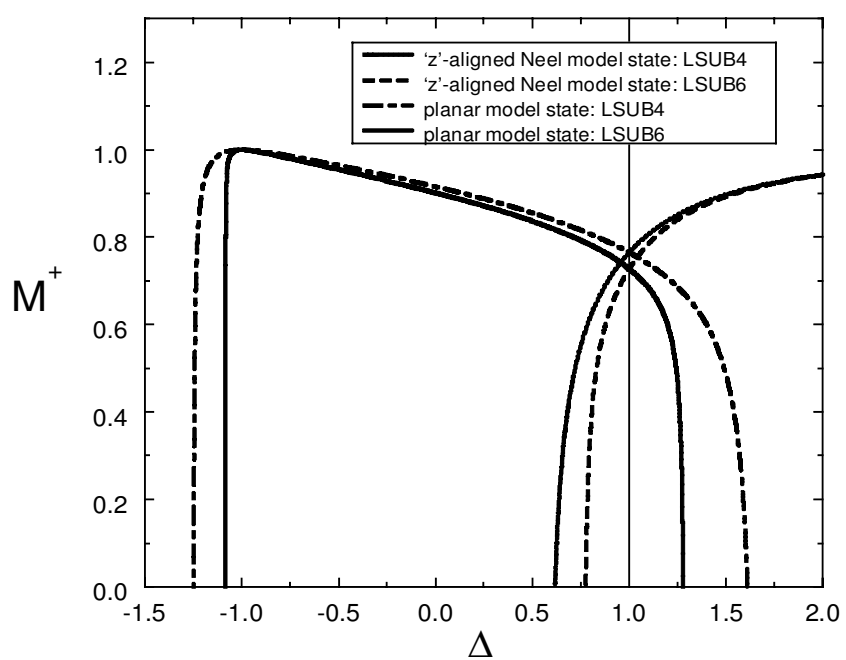

Figure 2. Results for the CCM sublattice magnetization $M^{+}$ of the spin- $-\frac{1}{2} X X Z$ model on the $2 \mathrm{D}$ square lattice using the $\mathrm{LSUB} m$ approximation with $m=4,6$ based on both the planar and $z$-aligned Néel model states. The results indicate non-zero long-range order in the $x y$ plane for $-1<\Delta<1$, and along the $z$ axis for $\Delta>1$.

in section 2, to give the LSUB $m$ estimate for $M^{+}$,

$$
\begin{aligned}
M^{+} & =1-2 \sum_{r=1}^{N_{F}} n_{r}\left(n_{r} !\right)^{2} v_{r} \tilde{x}_{r} \chi_{r} \\
& =1-2 \sum_{r=1}^{N_{F}} n_{r}\left(n_{r} !\right) \tilde{x}_{r} x_{r},
\end{aligned}
$$

where $n_{r}$ is the number of spin flips with respect to $|\Phi\rangle$ for the $r$ th fundamental configuration, and where we have introduced the notation $x_{r} \equiv \chi_{r}$ and $\tilde{x}_{r} \equiv$ $\left(n_{r} !\right) v_{r} \tilde{x}_{r}$. For the square lattice considered here $v_{r}=4$ or 8 for all allowed fundamental configurations.

Evaluation of the sublattice magnetization requires both the ket- and bra-state cluster correlation coefficients. We have described the computation of the ketstate coefficients in broad outline above. The bra-state coefficients are calculated similarly, by making use of the generic linear equations (9), once the ket-state coefficients are known. The actual procedure is straightforward, and is also described in more detail elsewhere [29]

Results for $M^{+}$for the spin- $\frac{1}{2} 2 \mathrm{D} X X Z$ model on the square lattice using both CCM model states are shown in figure 2. Table 1 also summarizes the results at the isotropic Heisenberg point, $\Delta=1$. We note again that the corresponding $\operatorname{LSUB} m$ results for $M^{+}$at a given truncation level $m$ are identical at $\Delta=1$. We see clearly that over the entire range $-1<\Delta<1$ our results seem to converge well to a non-zero in-plane long-range order, with a non-zero planar sublattice magnetization, whereas for all $\Delta>1$ we have comparable non-zero 
long-range order along the spin $z$ axis and a non-zero $z$ component of sublattice magnetization.

We also note the divergence in $M^{+}$near the critical points $\Delta_{\mathrm{A}}^{z}$ and $\Delta_{\mathrm{A}}^{\mathrm{p}}$, which strongly reinforces our interpretation of these points as reflecting a phase transition. As we approach one of these antiferromagnetic critical points for a given LSUB $m$ approximation, we typically find that at least one of the CCM correlation coefficients $x_{r}$ becomes very large, and hence $M^{+}$diverges from equation (36).

We once again attempt a simple heuristic extrapolation of our LSUB $m$ results for $M^{+}$at the Heisenberg point $(\Delta=1)$ to the limit $m \rightarrow \infty$, in order to compare our results with those from other calculations. As has been found elsewhere [11] the results for $M^{+}$extrapolate well to their asymptotic value with a leading correction that scales as $m^{-1}$. As shown in table 1 we thus obtain an extrapolated value, $M^{+}=0.62$. This compares extremely well with the best available Monte Carlo simulation value of Runge [32] $M^{+}=0.615 \pm 0.005$, and with the value $M^{+}=0.62 \pm 0.02$ from series expansion techniques [34]

\subsection{Critical properties}

We have demonstrated so far that CCM calculations using the LSUB $m$ approximation scheme based on different model states are well able to describe both the Ising-like phase for $\Delta>1$ and the planar-like phase for $-1<\Delta<1$ of the spin- $\frac{1}{2} 2 \mathrm{D} X X Z$ model on the square lattice. The results are not only extremely accurate for such quantities as the ground-state energy and sublattice magnetization as functions of $\Delta$, but calculations based on each model state separately give accurate results for the critical values $\Delta_{\mathrm{c}}$ which delimit the regime in $\Delta$ in which that model state is apposite. These critical values clearly mark (at the level of approximation considered) the physical phase boundaries. In this context it is natural to ask now whether our CCM formalism also has the power to predict the critical behaviour of the physical observables near such phase transitions, i.e. whether we can extract from the LSUB $m$ results useful information on critical indices. We show below, by specific application to the same spin- $\frac{1}{2}$ square-lattice $X X Z$ model, that this question can firmly be answered in the affirmative.

Thus, the critical index for the singular (non-analytic) term in $E_{\mathrm{g}} / N$ near an LSUB $m$ critical point $\Delta_{\mathrm{c}}(m)$ can first be obtained, for example, by direct examination of the anisotropy susceptibility, $\chi_{\mathrm{a}} \equiv-\partial^{2}\left(E_{\mathrm{g}} / N\right) / \partial \Delta^{2}$, of equation (34). For $m>2$ we find,

$$
\chi_{\mathrm{a}}^{m}(\Delta)-\rightarrow \bar{\chi}_{\mathrm{a}}^{m}\left|\Delta-\Delta_{\mathrm{c}}(m)\right|^{-\alpha_{0}} ; \quad \Delta \rightarrow \Delta_{\mathrm{c}}(m) .
$$

Direct calculation for the LSUB $m$ approximations using both the $z$-aligned and planar Néel model states shows that for $m>2$ we have $\alpha_{0} \approx 1.500 \pm 0.005$. However, the prefactors $\bar{\chi}_{\mathrm{a}}^{m}$ in equation (38) are themselves strongly dependent on the truncation index $m$. We may now use a variant of the so-called coherent anomaly method (CAM) of Suzuki [35] to extract further information. Thus, we attempt to fit $\bar{\chi}_{\mathrm{a}}^{m}$ with the coherent anomaly form,

$$
\bar{\chi}_{\mathrm{a}}^{m} \rightarrow K\left|\Delta_{\mathrm{c}}(\infty)-\Delta_{\mathrm{c}}(m)\right|^{v} ; \quad \Delta \rightarrow \Delta_{\mathrm{c}}(\infty),
$$

where $K$ is a constant. Thus, as explained by Suzuki [35] one may intuit or prove that the exact $\chi_{\mathrm{a}}(\Delta)$ has the critical form,

$$
\chi_{\mathrm{a}}(\Delta)-\rightarrow \kappa\left|\Delta-\Delta_{\mathrm{c}}(\infty)\right|^{-\alpha_{0}+v} ; \quad \Delta \rightarrow \Delta_{\mathrm{c}}(\infty) \equiv \Delta_{\mathrm{c}},
$$

where $\kappa$ is a constant.

A CAM analysis along these lines of the LSUB $m$ results based on the $z$-aligned Néel state gives $v \approx 1.25$ using the $\Delta_{\mathrm{A}}^{z}(4)$ and $\Delta_{\mathrm{A}}^{z}(6)$ data, and $v \approx 0.97$ using the $\Delta_{\mathrm{A}}^{z}(6)$ and $\Delta_{\mathrm{A}}^{z}(8)$ data. We thus obtain a singular term in $E_{\mathrm{g}} / N$ near $\Delta_{\mathrm{A}}^{z}$ with a critical exponent $2-\alpha_{0}+v \approx$ 1.50-1.75. This may be compared with the corresponding value of $3 / 2$ for both the mean-field-like CCM SUB2 approximation (in which all 2-spin-flip correlation terms are retained, however far apart on the lattice) and linear spin-wave theory (LSWT). A corresponding analysis may also be carried out on the LSUB $m$ results based on the planar model state near $\Delta_{\mathrm{A}}^{\mathrm{p}}$. We again find $\alpha_{0} \approx 1.500 \pm 0.005$ for both the LSUB4 and LSUB6 results, and a corresponding critical anomaly based on these of $v \approx 1.27$. We thus obtain a singular term in $E_{\mathrm{g}} / N$ near $\Delta_{\mathrm{A}}^{\mathrm{p}}$ with a critical exponent $2-\alpha_{0}+v \approx 1.77$. These preliminary data are clearly compatible with the hypothesis that the critical exponent in the energy is the same on both sides of the antiferromagnetic phase transition $\Delta_{A}$.

Similar CAM analyses can also be performed for the ground-state energy near the corresponding ferromagnetic critical point $\Delta_{\mathrm{F}}^{\mathrm{p}}$, and for such other properties as the sublattice magnetization $M^{+}$near any of these critical points.

\section{Conclusions}

Our aim in the present paper has been to show that the well-known and much used coupled cluster method of microscopic quantum many-body theory may be used to great advantage to study quantum spin-lattice models at a level which includes the quantitative identification of zero-temperature quantum phase transition points between states of different magnetic ordering, and the various critical indices which describe their behaviour in the vicinity of these transitions. The CCM is now in the position of being virtually the only fully microscopic method available which can yield very ac- 
curate results for both local ground-state properties and the global critical behaviour of these highly non-trivial systems.

We note that for spin-lattice models with nearestneighbour interactions on bipartite lattices, such as the $X X Z$ model considered here, quantum Monte Carlo results are also available. These are practicable for these models since the infamous 'minus-sign problem' which bedevils Monte Carlo simulations can be circumvented by mapping the models onto equivalent bosonic problems or, equivalently, by utilizing the Marshall sign rule [36] concerning the phase relations between the projection coefficients of the ground-state wave function onto a complete set of spin configurations. We have seen that our own CCM formalism can now be effectively implemented computationally to levels where the results are comparable in accuracy to the best Monte Carlo results for these cases.

By contrast, for frustrated models (e.g. models with both nearest-neighbour and next-nearest-neighbour interactions $[12,24,25]$ or the same $X X Z$ model considered here but on a 2D triangular lattice [13]) Monte Carlo simulations are much more difficult to implement (e.g. see [37] for the typical case of the isotropic spin- $\frac{1}{2}$ Heisenberg model on a triangular lattice). On the other hand, as we have shown elsewhere [12, 13, 24, 25] the CCM is no more difficult to implement, either in principle or in practice, for such frustrated systems. In particular, there is now a real hope that the CCM results can be used to guide more ambitious fixed-node-type Monte Carlo simulations of such systems, by providing better trial wave functions whose nodal surface structure can be taken from CCM LSUB $m$ results.

Further extensions of our CCM formalism may also be envisaged. For example, LSUB $m$ calculations on spin lattices where the spins have quantum number $s>\frac{1}{2}$ present no real difficulty [10(f)] Thus, one merely generalizes to the case of 'multiple occupancy' of the sites $\left\{i_{1}, \ldots, i_{n}\right\}$ in the retained fundamental configurations, where each site can now be 'occupied' up to $2 s$ times (i.e. since the spin-raising operator $s_{k}^{+}$on site $k$ can act on the 'ferromagnetic' model state $|\Phi\rangle$ in the local spin coordinates up to $2 s$ times before it annihilates $|\Phi\rangle)$. We are also hopeful that our general methodology can be applied to other unconventional (non-Fermi-liquidtype) systems of the sort discussed in section 1. Systems which seem particularly ripe for further application of the CCM techniques considered here include the valence-bond solids $[24,26]$ and lattice models with electronic degrees of freedom, such as the Hubbard model [17, 27].

Finally, we note that it would be of great interest to extend the CCM treatment of all of the above models, including the $X X Z$ model considered here, to finite tem- peratures. One obvious way to do this would be to extend the formalism by utilizing the general framework of thermofield dynamics [38] In the same context we also note that Mukherjee [39] and others have suggested alternative ways to extend the CCM to incorporate a thermal averaging procedure.

We thank J. B. Parkinson, Y. Xian, and C. Zeng for useful discussions. One of us (R.F.B.) also gratefully acknowledges a research grant from the Engineering and Physical Sciences Research Council (EPSRC) of Great Britain.

\section{References}

[1] Coester, F., 1958, Nucl Phys., 7, 421; Coester, F., and Kümmel, H., 1960, ibid., 17, 477.

[2] Cižex, J., 1966, J. chem. Phys., 45, 4256; 1969, Adv. chem. Phys., 14, 35.

[3] Bishop, R. F., and Lührmann, K. H., 1978, Phys. Rev. B, 17, 3757.

[4] Kümmel, H., Lührmann, K. H., and Zabolitzky, J. G., 1978, Phys. Rep., 36C, 1.

[5] Arponen, J. S., 1983, Ann. Phys. (New York), 151, 311.

[6] Bishop, R. F., and Kummel, H., 1987, Phys. Today, 40(3), 52.

[7] Arponen, J. S., Bishop, R. F., and Pajanne, E., 1987, Phys. Rev. A, 36, 2519; 1987, ibid., 36, 2539; 1987, Condensed Matter Theories, Vol. 2, edited by P. Vashishta, R. K. Kalia and R. F. Bishop (New York: Plenum), p. 357.

[8] Bartlett, R. J., 1989, J. phys. Chem., 93, 1697.

[9] Bishop, R. F., 1991, Theor. Chim. Acta, 80, 95.

[10] (a) Bishop, R. F., Parkinson, J. B., and Yang Xian, 1991, Phys. Rev. B, 43, 13 782; (b) 1991, ibid., 44, 9425; (c) 1992, ibid. , 46, 880; (d) 1991, Theor. Chim. Acta, 80, 181; (e) 1992, J. Phys.: Condens. Matter, 4, 5783; (f) 1993, ibid., 5, 9169.

[11] Bishop, R. F., Hale, R. G., and Xian, Y., 1994, Phys. Rev. Lett., 73, 3157.

[12] Farnell, D. J. J., and Parkinson, J. B., 1994, J. Phys.: Condens. Matter, 6, 5521.

[13] (a) Zeng, C., Staples, I., and Bishop, R. F., 1995, J. Phys.: Condens. Matter, 7, 9021; (b) 1996, Phys. Rev. B, 53, 9168.

[14] Bishop, R. F., Farnell, D. J. J., and Parkinson, J. B., 1996, J. Phys.: Condens. Matter, 8, 11153.

[15] Bishop, R. F., and Xian, Y., 1994, Condensed Matter Theories, Vol. 9, edited by J. W. Clark, K. A. Shoaib and A. Sadiq (Commack, New York: Nova Science Publ.), p. 433.

[16] Bishop, R. F., 1995, Recent Progress in Many-Body Theories, Vol. 4, edited by. E. Schachinger, H. Mitter and H. Sormann (New York: Plenum), p. 195.

[17] Bishop, R. F., Xian, Y., and Zeng, C., 1995, Int. J. quantum Chem., 55, 181.

[18] Bishop, R. F., and Xian, Y., 1993, Acta Phys. Polonica B, 24, 541; Bishop, R. F., Kendall, A. S., Wong, L.Y., and Yang Xian, 1993, Phys. Rev. D, 48, 887; Bishop, R. F., and XIan, Y., 1994, Nucl. Phys. B (Proc. Suppl.), 34, 808; Davidson. N. J. and Bishop, R. F., 
1995, ibid., 42, 817; Baker, S. J., Bishop, R. F. and Davidson, N. J., 1997, ibid., 53, 834.

[19] Roger, M., and Hetherington, J. H., 1990, Phys. Rev. B, 41, 200.

[20] Lo, C. F., Pang, K. K., and Wang, Y. L., 1991, J. Appl. Phys., 70, 6080.

[21] Harris, F. E., 1993, Phys. Rev. B, 47, 7903.

[22] Cornu, F., Jolicoeur, Th., and Le Guillou, J. C., 1994, Phys. Rev. B, 49, 9548.

[23] Wong, W. H., Lo, C. F., and Wang, Y. L., 1994, Phys. Rev. B, 50, 6126.

[24] Xian, Y., 1994, J. Phys.: Condens. Matter, 6, 5965.

[25] Bursill, R., Gehring, G. A., Farnell, D. J. J., Parkinson, J. B., Xiang, T., and Zeng, C., 1995, J. Phys.: Condens. Matter, 7, 8605.

[26] Xian, Y., 1995, Condensed Matter Theories, Vol. 10, edited by M. Casas, M. de Llano and A. Polls (Commack, New York: Nova Science Publ.), p. 541.

[27] Roger, M., and Hetherington, J. H., 1990, Europhys. Lett., 11, 255; Lo, C. F., Manousakis, E., and Wang, Y. L., 1991, Phys. Lett. A, 156, 42; Petit, F., and Roger, M., 1994, Phys. Rev. B, 49, 3453.

[28] Llewellyn Smith, C. H., and Watson, N. J., 1993, Phys. Lett. B, 302, 463.
[29] Zeng, C., Farnell, D. J. J., and Bishop, R. F., 1998, J. statist. Phys., 90, 327.

[30] Barnes, T., Kotchan, D., and Swanson, E. S., 1989, Phys. Rev. B, 39, 4357.

[31] Kubo, K., and Kishi, T., 1988, Phys. Rev. Lett., 61, 2585.

[32] Runge, K. J., 1992, Phys. Rev. B, 45, 12 292; 1992, ibid., 45, 7229.

[33] Anderson, P. W., 1952, Phys. Rev., 86, 694; Oguchi, T., 1960, ibid., 117, 117.

[34] Singh, R. R. P., 1989, Phys. Rev. B, 39, 9760; Singh, R. R. P., and Huse, D. A., 1989, ibid., 40, 7247; Zheng, W., Oitmaa, J., and Hamer, C. J., 1991, ibid., 43, 8321.

[35] Suzuki, M., 1986, J. Phys. Soc. (Japan), 55, 4205.

[36] Marshall, W., 1955, Proc. R. Soc. (London) A, 232, 48.

[37] Boninsegni, M., 1995, Phys. Rev. B, 52, 5304.

[38] Umez awa, H., Matsumoto, H., and Tachiki, M., 1982, Thermo Field Dynamics and Condensed States (Amsterdam: North-Holland); UMez Awa, H., and Yamanaka, Y., 1988, Adv. Phys., 37, 531.

[39] Sanyal, G., Mandal, S. H., and Mukherjee, D., 1992, Chem. Phys. Lett., 192, 55. 